\title{
Vector Magnetic Fields at the Base of Filaments and the Filament Environment
}

\author{
Jingxiu Wang and Wei $\mathrm{Li}$ \\ Beijing Astronomical Observatory, Chinese Academy of Sciences, \\ Beijing 100080, China
}

\begin{abstract}
Based on an analysis of three active filaments in AR 6891, we find that vector magnetic fields at the base of filaments and the filament environment is characterized by the following: (1) The transverse field is parallel along the magnetic neutral line for most of the filaments. The average transverse field beneath the filaments is more than $400 \mathrm{G}$. (2) The horizontal gradient of the line-of-sight field crossing the neutral line is, more or less, constant along the major fraction of a filament, but very steep at the two ends. The average gradient is $0.06 \mathrm{G} / \mathrm{km}$. (3) For each of the filaments there is a squeezing magnetic structure which represents an intrusion of a satellite bipole into the main flux system. The neutral line for a squeezing magnetic structure has a large curvature, a strong sheared transverse field of more than $1 \mathrm{KG}$, and a steep field gradient of approximate $0.3 \mathrm{G} / \mathrm{km}$. (4) The transverse field and field gradient are clearly enhanced before the filament eruption.
\end{abstract}

\section{Introduction}

Almost 10 years ago, Leroy (1989) stressed the urgent need of better knowledge of the photospheric magnetic neutral line hosting a filament. So far the situation he mentioned remains that the photospheric field around the neutral line is rather poorly known. As he emphasized, to learn the actual magnetic structure of the neutral line is a compulsory step to improving our understanding of the filament magnetic field.

In this report we present the first effort made at the Huairou station of Beijing Astronomical Observatory to determine the actual structure of the vector magnetic field under the filament and the filament environment. The sample we chose for this study consists of three active filaments in active region (AR) 6891 in October 1991. All three filaments erupted after hours of evolution.

The data base for this study is vector magnetograms taken in the FeI $5324 \AA$ line and $H \beta$ filtergrams. The spatial resolution for the data is about 2 arcsec, the temporal resolution is minutes for filtergrams, tens of minutes for vector magnetogram. The noise level is less than $20 \mathrm{G}$ for the line-of-sight magnetogram, and $150 \mathrm{G}$ for the transverse field measurement. The calibration and data reduction have been described by Wang et al. (1996). 


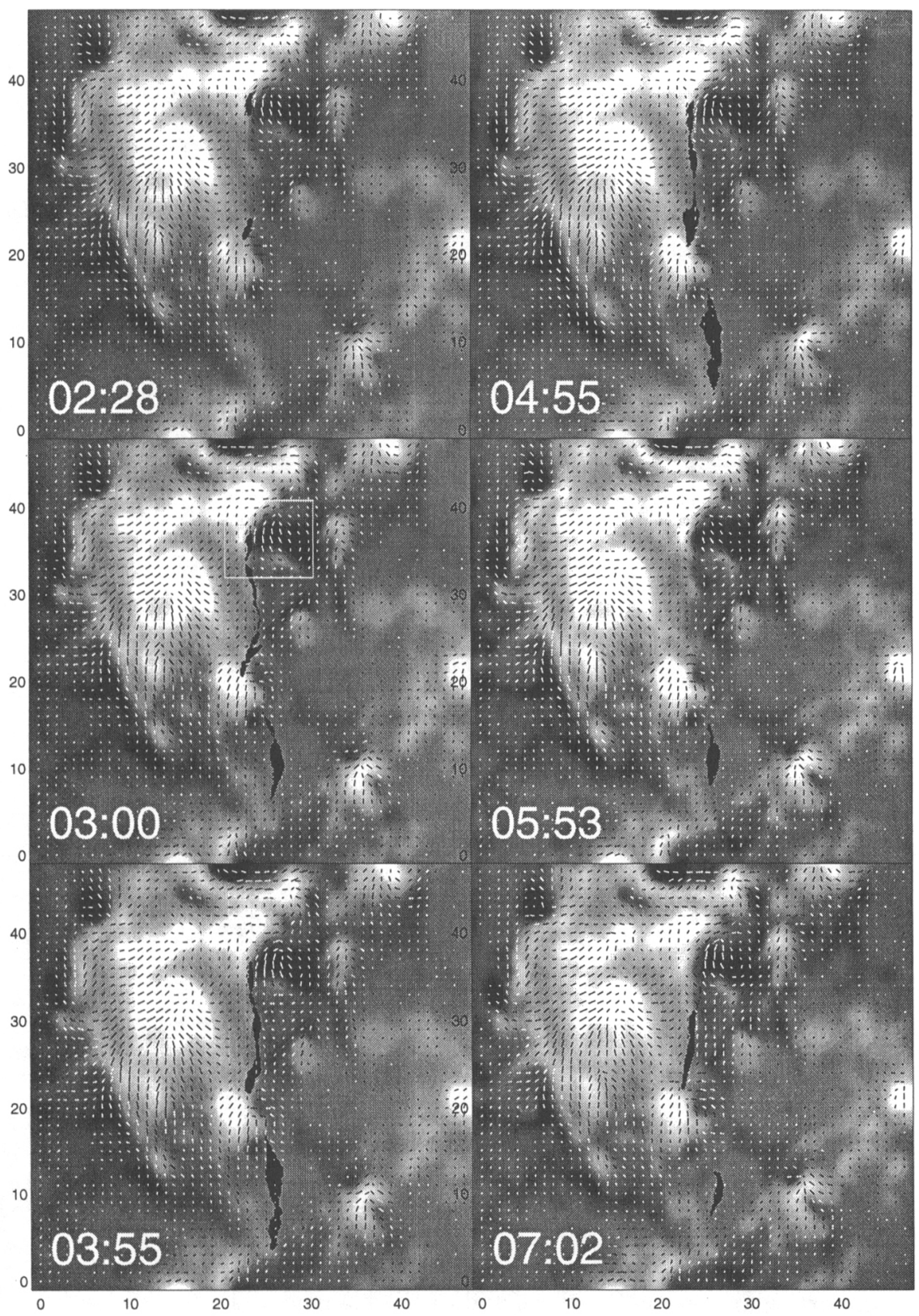

Figure 1. Time sequence of vector magnetograms superposed on the filament morphology observed at the same time. 


\section{Data Analyses and Results}

Figure 1 is a time sequence of vector magnetograms superposed on the $H^{*} \beta$ filament as dark fringes for the filament on October 27, 1991 (Filament 1). This filament consists of two pieces, one active piece located in the northern part (top of the figure) and the quiet piece in the southern part. The line-of-sight field is shown by brighter (darker) patches for positive (negative) polarity. The transverse field is shown by bars with length proportional to the field strength, and orientation parallel to the field direction with a $180 \mathrm{deg}$. ambiguity.
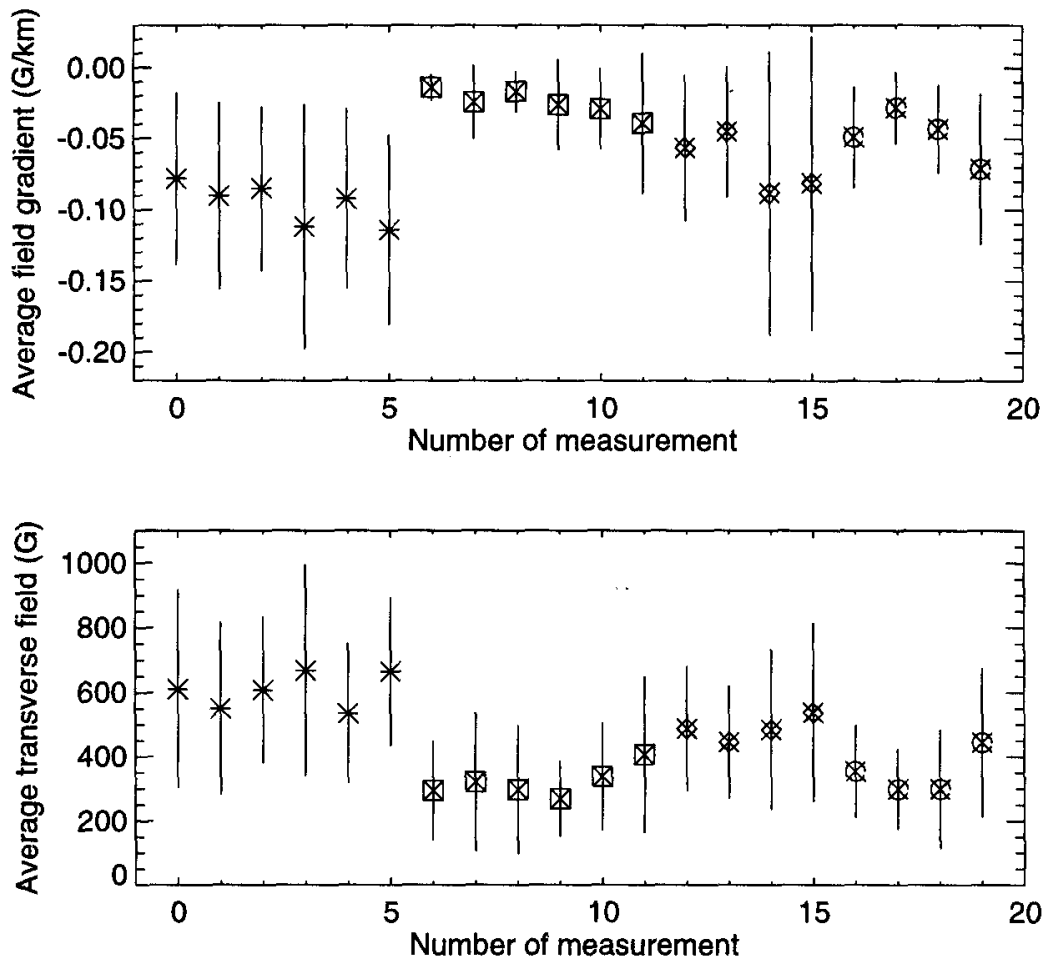

Figure 2. Measurements of average field gradient and transverse field. Asterisks and squares are for the active and quiet pieces of Filament 1, respectively; diamonds for Filament 2; circles for Filament 3.

It is unexpected that the transverse field beneath the filament is rather strong, particularly at the northern end of the filament. The transverse field is parallel to the magnetic neutral line over most of the filament. The active piece of filament ends in the south where the transverse field runs across the neutral line. The average transverse field is $600 \mathrm{G}$ along the active piece of the filament and $300 \mathrm{G}$ for the quiet piece (see Figure 2 for details). It is astonishing to see that at the northern end, the transverse field is more than $1 \mathrm{KG}$, and exactly parallel to the curved neutral line. The general pattern of transverse field for two 
other filaments on October 28 and 30 (Filament 2 and 3 ) is the same as described above, but the field strength varies among different filaments (see Figure 2).

The horizontal gradient of the line-of-sight field crossing the neutral line (referred to as field gradient) is measured within a distance of 4 arcsec from positive to negative polarity. The field gradient for the centralized major fraction of a filament is almost constant, approximately 0.06 to $0.07 \mathrm{G} / \mathrm{km}$. However, it is very steep at the two filament ends, at least $0.3 \mathrm{G} / \mathrm{km}$.

A common element of the vector field structure of all three filaments is a characteristic field pattern which we called as a squeezing magnetic structure. It consists of two flux patches which come from different flux systems, with one flux patch squeezing into the other, forming a curved neutral line. The neutral line shows a strong sheared transverse field of more than $1 \mathrm{KG}$, and a steep field gradient of approximately $0.3 \mathrm{G} / \mathrm{km}$. The impacting flux comes from a newly emerging satellite bipole. An example of squeezing structure is shown in the small box in Figure 1 for Filament 1.

In Figure 2 we summarize all the measurements for these three filaments. The measurements at different times show the vector field evolution which is associated with the destablization of the filament. The last measurement for each filament is referred to as the time of filament eruption. The tendency is clear that the transverse field and field gradient are obviously enhanced before the filament eruption.

\section{Summary}

We have explored the vector field structure beneath filaments and the filament environment for the first time. It is characterized by a strong sheared transverse field and a certain range of field gradient. The squeezing magnetic structure at the far end of each filament is identified to be a common element for all three filaments.

We note that the filaments studied can be classified to be of A-type magnetic boundary (Tandberg-Hanssen 1974), i.e., they form within an active region. However, they do appear to separate the main flux system and the satellite bipoles. Thinking about Tang's (1987) discovery, we tentatively suggest that all filaments may, in fact, represent an environment of the interface of different sources of flux systems.

Acknowledgments. The paper was partly written when J. Wang visited Meudon Observatory. He thanks Dr. B. Schmieder for kind hospitality.

\section{References}

Leroy, J-L. 1989, in Dynamics and Structure of Quiescent Solar Prominences, E.R. Priest (ed.), Kluwer Acad. Publ., Dordrecht, Holland, p. 77

Tang, F. 1987, Sol. Phys., 107, 233

Tandberg-Hanssen, E. 1974, Solar Prominences, D. Reidel, Dordrecht, Holland

Wang, J., Shi, Z., Wang, H. and Lü, Y. 1996, ApJ, 456, 861 